\title{
Mixing parameters for geometry optimization using the Hamiltonian algorithm
}

\author{
Hiroyuki Teramae, ${ }^{* 1}$ Takayoshi Ishimoto, ${ }^{2}$ and Umpei Nagashima ${ }^{3}$ \\ ${ }^{1}$ Department of Chemistry, Faculty of Science, Josai University, 1-1 Keyakidai, \\ Sakado, Saitama 350-0295, Japan \\ teramae@josai.ac.jp \\ ${ }^{2}$ INAMORI Frontier Research Center, Kyushu University, 744 Motooka, Nishi- \\ ku, Fukuoka 819-0395, Japan \\ ${ }^{3}$ Research Institute for Computational Science, National Institute of Advanced \\ Industrial Science and Technology, 1-1-1 Umezono, Tsukuba, Ibaraki 305-8568, \\ Japan
}

\begin{abstract}
We study the mixing parameters for the search of an optimal geometry using the Hamiltonian algorithm (HA) combined with ab initio molecular orbital calculations. We choose the dihedral angle -C-C-C-C- of the butane molecule as an example. HF/3-21G level calculations are employed as the molecular orbital calculations. The distributions of the eigenvalues of mixing coefficients are fitted with the linear, quadratic, and quartic functions. Analyses of HA calculations both up to 2000 and 60000 iterative calculations show a possibility that the mixing process reduces the number of iterations.
\end{abstract}

Keywords: Hamiltonian algorithm, mixing, molecular orbital, molecular dynamics 


\section{Introduction}

Over the last two decades of the 20th century, the energy gradient method combined with the ab initio molecular orbital theory has been widely used and has enabled the optimization of a molecular structure with electronic structure calculations. In such optimization, several optimization methods, such as the Newton-Raphson, steepest descent, and conjugate gradient methods, were usually applied as algorithms of the optimization itself. These methods, however, have two difficulties that should be overcome in searching for the global minimum.

(1) It is necessary to start from the vicinity of minima or the optimization process sometimes diverges.

(2) Starting from one minimum point, it is impossible to locate another minimum point, i.e., it is impossible to escape from a local minimum.

For example, Teramae and coworkers accidentally found an "ortho" conformation rather than "anti" and "gauche" conformations for the saturated carbon chain $\mathrm{C}_{4} \mathrm{Y}_{10}$ [1,2]. The ortho conformation had not been considered except for the case that $\mathrm{Y}$ is fluorine, $\mathrm{C}_{4} \mathrm{~F}_{10}$. Even in $\mathrm{C}_{4} \mathrm{~F}_{10}$, the "ortho" structure had not been studied in detail.

The Monte Carlo (MC) method, as well as the simulated annealing method, is usually applied to escape from a local minimum. In the MC method, a large part of the computational time is wasted in calculating an unstable structure of higher energy. In the simulated annealing method, it is difficult to control the temperature of the critical point. Optimization using the above two methods combined with $a b$ initio molecular orbital calculations is considered difficult and has not been reported to the authors' best knowledge.

The Hamiltonian algorithm (HA) [3] is proposed as a general optimization method and was applied to solve many problems, such as the optimization of packet routing, the optimization of axis adjustment of optical fibers, and the optimization of the quantum table used by the Joint Photographic Experts Group (JPEG) [4], and a design theory of material systems [5]. We have recently applied the HA to solve the quantum chemistry problem [6], i.e., the optimization of molecular structures. We showed that the HA enables the effective search of the potential energy surface and that we can find an energy minimum even if we start from another energy local minimum by determining the optimal structure of the HCN molecule starting from the HNC molecule. We also performed the molecular dynamics studies on the glycine oligomers [7, 8], the tri-peptides (glycine, alanine, valine, leucine, and isoleucine trimers) with ab initio molecular orbital calculations [9] and enkephalin with the empirical force field [10].

For a more advanced study, the HA has recently been employed to optimize the molecular structure of a benzodiazepine minor tranquilizer and showed the possibility of designing a drug [11].

The optimized structures themselves are easily obtained for relatively small molecules if we employ the HA by choosing suitable "mixing parameters" [6-11]. The mixing parameters are considered to represent an approximate incorporation of the mixing of the statistical physics.

In the present article, we study the behavior of the molecular dynamics with the Hamiltonian algorithm, especially an effect of the mixing parameters. For its purpose, we select the butane molecule and pay an attention on the torsional degree of freedom. The molecular dynamics calculations are performed with all of the degrees of freedom. Although the diatomic molecule has just one degree of freedom, we consider the diatomic molecule is too simple for an example. We study the effect of choosing a mixing parameter and the eigenvalues of the matrix representing the mixing of the motion of an atomic nucleus. 


\section{Method of Calculation}

The details of the HA have been discussed elsewhere. In this article, we only indicate the part considered necessary for the present study.

In the HA, we consider the virtual motion of particles $x$ (in this case, the atomic nuclei) with the cost function $V(x)$, which is the energy calculated by the $a b$ initio molecular orbital method in the present case, and develop a Hamiltonian of the motion

$$
H(p, x)=\frac{1}{2} \sum_{i, j} \frac{p_{i} b_{i j} p_{j}}{\sqrt{m_{i} m_{j}}}+V(x),
$$

where $\left(b_{i j}\right)$ is a positive definite symmetric matrix, $p_{i}$ is momentum, and $m_{i}$ is the mass of particles. The first term on the right-hand side of the equation is the virtual kinetic energy term, and we allow the off-diagonal term of the kinetic energy. The off-diagonal term reveals the mixing of the motion of each particle, and the coefficient $b_{i j}$ represents the degree of this mixing. This mixing procedure enables the effective search of the energy surface.[7]

By including the nondiagonal term of the kinetic energy, the randomness of the motion would be expected to increase and the dependence of the motion on the initial structure would be expected to decrease. The possibility to reach the global minimum thus becomes larger, which is clearly shown in the optimization of axis adjustment of optical fibers. It is equivalent to ab initio molecular dynamics simulation when the off-diagonal terms are zero. The usual molecular dynamics (MD) calculation depends on the initial structures and requires us to start from several initial structures, increasing the total computational time.

The following is used to define the coefficient $b_{i j}$. Let $\mathbf{B}$ denote the positive definite symmetric matrix $\left(b_{i j}\right)$.

$$
\mathbf{D}=\mathbf{I}+\lambda \mathbf{A},
$$

where $\mathrm{I}$ is an identity matrix. In order to avoid an incorporation of the regularity, matrix $\mathbf{A}$ is produced by random number generator as followings.

$$
A_{i j}=A_{j i}=\left(a_{i j}-\frac{1}{2}\right)
$$

Where $a_{i j}$ is a random number between 0 and 1 . We set $\lambda=\frac{1}{2}$ in eq. (2).

The matrix $\mathbf{C}$ is obtained from the matrix $\mathbf{D}$ by using the Gram-Schmidt process. The positive definite symmetric matrix $\mathbf{B}$ is given by using the nonconstant eigenvalue $\varepsilon$

$$
\mathbf{B}=\mathbf{d} \mathrm{C}^{\mathrm{T}} .
$$

where $\boldsymbol{\varepsilon}$ is a diagonal matrix, whose diagonal elements are $\left\{\varepsilon_{i}\right\}$

Here, we just note that the degree of mixing is represented by the eigenvalues of the matrix. We define the maximum difference between the eigenvalues of the matrix $\mathbf{B}$ and 1 as the mixing coefficient. The eigenvalues were previously given by a linear function. However, in the present paper, we also try to provide them using a quadratic function and a quartic function, as shown in Fig. 1. The i-th eigenvalue is, therefore, given in the following equation, where $\mathrm{N}$ is the number 
of atoms, i runs from 0 to $3 \mathrm{~N}-1, \mathrm{~L}$ is 1 (linear), 2 (quadratic) or 4 (quartic), and $\Delta(0 \leq \Delta \leq 1)$ is a mixing coefficient.

$$
\begin{aligned}
& \varepsilon_{i}=1+\Delta \times\left(\frac{2 i-3 N}{3 N-1}\right)^{\frac{1}{L}} \quad(2 i-3 N \geq 0) \\
& \varepsilon_{i}=1-\Delta \times\left(-\frac{2 i-3 N}{3 N-1}\right)^{\frac{1}{L}} \quad(2 i-3 N<0)
\end{aligned}
$$

The power $(1 / \mathrm{L})$ is defined because we used the fitting function of the form like $\mathrm{x}=\mathrm{y}^{\mathrm{L}}$ as shown in Fig. 1.

The equation of motion is presented as

$$
\ddot{x}_{i}=\sum_{j} \frac{b_{i j} f_{j}}{\sqrt{m_{i} m_{j}}},
$$

where $f_{i}$ is the force acting on the atom $i$, which can be calculated by the energy gradient method. Under a certain initial structure and an initial kinetic energy, we can successively solve the equation of motion by the Verlet method $[8,9]$ while keeping the total energy of the system constant. When the constraints of the kinetic energies for the translations of the center of gravity are zero,

$$
\sum_{i} m_{i} v_{i}(0)=0 \text {. }
$$

The initial geometry and velocity should be given as the parameters of the motion. We examined the initial kinetic energy in 0.05 a.u. in this study. We do not vary only the torsional angle, but all the degree of freedom of butane molecule. The kinetic energy is almost 10 times of the torsional barrier height, however, the kinetic energy is distributed to all other degree of freedom, 3N-7. Therefore, the kinetic energy for torsional barrier is not so large. If we increase kinetic energy, the molecule usually will move more rapidly or sometimes will decompose to its substructures. The kinetic energy of the present study is indeed too small for the purpose of the geometry optimization, because the kinetic energy was selected in order to see clearly the difference of the mixing parameters.

The time interval $\Delta t$ is 40 a.u. throughout the present study. We keep both the geometry and potential energy (= cost function) of the molecule during the motion period, and determine the minimum point after all of the calculations have been finished. We can find the minimum of the potential energy and perform the optimization of the molecular structure, if the motion exhibits mixing and we can observe the motion for a sufficient long time.

The optimization procedure utilizes classical dynamics and, therefore, we call it a "Hamiltonian algorithm". We developed a program based on the GAMESS program [10]. The RHF calculation with the $3-21 \mathrm{G}$ basis set [11] is used throughout. The time step used to solve the Verlet method is 40 a.u., as denoted above, and the iterative calculations are repeated 2000 and 60000 times. All of the calculations start from the HF/3-21G optimized trans structure obtained by using the Gaussian03 [12] program. 


\section{Results and Discussion}

Figure 2 shows the plots of the dihedral angles of the C-C-C-C backbone in the butane molecule for the first 2000 iterative HA calculations using the distribution of eigenvalues with linear, quadratic, and quartic functions. Figures 2 also shows a comparison between the results of the mixing coefficient 0.0 (i.e., no mixing) and those of the mixing coefficients 0.05 and 0.1 .

Regardless of the function type used, the graph clearly shows that the inclusion of the mixing coefficients 0.05 and 0.1 shifts the top of the graph to the left. This characteristic of the graph is clearest when using the quartic function as the distribution of the eigenvalues of the mixing matrix. In other words, the mixing reduces the cycle time of the vibration of the dihedral angle, although the dihedral angle itself seems to vacillate periodically as shown in Fig. 2. This result suggests a possibility that the mixing process reduces the number of iterations.

This effect is larger when the mixing parameter is 0.1 rather than when the mixing parameter is 0.05 . This result indicates that a larger mixing coefficient gives a larger effect. However, in the present study, the calculations with mixing coefficients larger than 0.15 almost show the divergence of the iterative HA calculations, particularly when using the quartic function. The larger mixing coefficient is expected to mix the motions of different coordinates strongly.

Figure 3 shows the plots of the dihedral angles obtained with full 60000 iterative HA calculations. The results are shown for four cases (a) without mixing and with mixing with (b) linear, (c) quadratic, and (d) quartic functions. The mixing coefficient is kept at 0.15 except for the case without mixing. The graphs (c) and (d) are terminated in the middle of the iterations because the iterative HA calculations diverge in the case of approximately 49000 calculations using the quadratic function and in the case of approximately 33000 calculations using the quartic function, suggesting that either the initial kinetic energy $0.05 \mathrm{a} . \mathrm{u}$. or the mixing coefficient 0.15 is very large in the cases of the quadratic and quartic functions.

In the case without mixing, the dihedral angles are kept at 180 degrees. This result shows that the butane molecule has been staying near the trans conformation during the HA calculations. In other cases with mixing, the dihedral angle of butane runs in full space, i.e., from 0 degrees to 360 degrees, after a certain number of iterative HA calculations depending on the distribution function of eigenvalues.

Note that, in the case of the linear function, the dihedral angle goes near the trans configuration in the case of approximately 38000 calculations and remains near the trans configuration in the case of approximately 10000 calculations. It deviates from the trans configuration and returns near the trans configuration in the case of approximately 57000 calculations, and remains near the trans configuration up to the end of the 60000 calculations. In the case of the quadratic function, the dihedral angle goes near the trans configuration in the case of approximately 34000 calculations and remains near the trans configuration up to 38000 calculations. It never returns near the trans configuration. In the case of the quartic function, the secondary phase indicating the return of the angle near the trans configuration is not observed or is too short to recognize.

Both Figs. 2 and 3 show a possibility that the mixing process reduces the number of iterations. Indeed, the gauche structures are appeared in Figs. 3b-3d. Listing only clear examples, the dihedral angles in Fig. 4b are around 300 degrees between 29000 and 32000 iterations and around 60 degrees between 33000 and 34000 iterations. The dihedral angles in Fig.3c are around 60 degrees between 29000 to 33000 iterations, and those in Fig.3d are around 60 degrees between 25500 and 27000 iterations. On the other hand, there is no gauche structure in Fig. 3a. 
Note that the results presented here are valid for the micro-canonical ensemble, however, we believe that the HA would have a possibility to be extended the canonical or NVT ensemble when the velocity Verlet method is employed.

About the comparison with the other optimization method, we found a result of a search for the $\mathrm{Lin}_{n}{ }^{0 /+1 / 1}(\mathrm{n}=5-7)$ lowest-energy structures using the ab initio gradient embedded genetic algorithm (GEGA)" by Alexandrova and Boldyrev [18]. In introduction section, we stated that it was impossible to escape from a local minimum using the optimization routines based on the Newton-Raphson method. However, very recent work showed that it is possible using a NewtonRaphson algorithm to go from one local minimum to another by optimizing in the presence of an applied external force. This procedure, termed enforced geometry optimization (EGO) has been successfully used to locate 10 different structural isomers starting from the converged geometry of cis-stilbene [19].

Maeda and Ohno proposed the global mapping method [20] and applied it to many systems, such as the potential surface searches of $\mathrm{H}_{2} \mathrm{O}$ and $\mathrm{H}_{2} \mathrm{CO}$ [20], $\mathrm{H}_{2} \mathrm{CO}$ and propyne [21], and $\mathrm{CH}_{3} \mathrm{CHO}$ [22], the minimal energy points on seams of crossing of $\mathrm{H}_{2} \mathrm{CO}$ [23], $\left(\mathrm{H}_{2} \mathrm{CO}\right)\left(\mathrm{H}_{2} \mathrm{O}\right)_{100}$ and $\mathrm{Si}_{6}\left(\mathrm{C}_{12} \mathrm{H}_{17}\right)_{6}$, in the $\operatorname{ONIOM}(\mathrm{QM}: \mathrm{MM})$ framework[24], and so on. It will be interesting to compare the results of the HA optimization with those of GEGA, EGO, and global mapping. This is, however, completely beyond the purpose of the present work and should be considered in near future. Furthermore, we consider the goals of these methods are a little bit different from the HA method. The HA method aims more global search of the molecular structures as will be shown below.

Recently, the multiple minimum problems of biomolecules in complex environments were extensively studied [25-36]. In order to show an example of the optimization of more realistic system than a butane molecule and the possibility of the application of the HA optimization on the biomolecule, the optimized HSD structures of $N$-acetyl L-histidine $N$-Methyl amide (NALHNMA), that was reported by Jalkannen et al. [32], are calculated by using the HA. Since discussing in detail about these results is completely beyond the purpose of the present study, we just would like to show the results. The optimization procedure used in these calculations is as follows. The 3000 iterative calculations using the HA is performed. The starting geometry is obtained by the usual geometry optimization by gausview [37] and Gaussian 09 (g09) [38] programs. We obtain snapshots at every 100 times calculations of 3000 HA calculations, and then switch to optimize with the conventional optimization procedure using g09 program [38] in order to obtain the rigorous structures. We obtain, therefore, 30 optimized structures for 3000 iterative calculations including duplicated structures. We usually obtain several isomers as shown below.

The HF/3-21G and PCM HF/3-21G optimization of the NALHNMA, and the NALHNMA with four water molecules were performed. The HF/3-21G results are shown in Table I, III, and IV, where the notation of the dihedral angles $\phi, \psi, \chi_{1}$, and $\chi_{2}$ are the same as the paper of Jalkanen et al[32]. The starting geometry corresponds to the last column of Table I and is found to be the least stable form among the structures obtained here. In this example, the HA can search the global minimum, even if the starting geometry is far from the global minimum.

The B3LYP/6-31G** structures of NALHNMA shown in Table II are obtained with the usual optimization from the HF/3-21G optimized structures and compared with the results of Jalkanen et al. The only global minimum is identical with that of their paper, and it is interesting that the remaining structures are completely different from each other. The potential surface of the NALHNMA is, indeed, very complex and there are many local energy minima.

The PCM B3LYP/aug-cc-pDVZ optimized structures of the NALHNMA and four water molecules are shown in Table V and Figs.4(a)-(e) where Fig.4(a) is 
calculated to be the most stable isomer. The Fig.4(a) and (b) as well as Fig.4 (d) and (e) has the same NALHNAM backbone, and the differences between them are just the direction of the water molecules. We obtained five structures reoptimizing the first five stable isomers in Table $\mathrm{V}$ using the standard optimization routines of g09. Although Jalkanen et al. did not show the detail of their optimized geometry and we could not show the detail, the five optimized geometries seem to be all different from that of Jalkanen et al. However, note that we did not perform the frequency analysis calculations because of the lack of our computational resources and these five minima may not the true minima.

In conclusion, we study the mixing parameters for the search of an optimal geometry using the Hamiltonian algorithm (HA) combined with ab initio molecular orbital calculations. We choose the dihedral angle $-\mathrm{C}-\mathrm{C}-\mathrm{C}-\mathrm{C}-$ of the butane molecule as an example. The distribution of the eigenvalues of mixing coefficients is fitted with the linear, quadratic, and quartic functions. Analyses of HA calculations both up to 2000 and 60000 iterative calculations show a possibility that the mixing process reduces the number of iterations.

The next step of the present study will be to apply the HA in larger molecules such as biomolecules, particularly protein molecules. We plan to try such a challenging calculation, the results of which will be published elsewhere. 


\section{References and note}

[1] Teramae H, Michl J (1994) Mol Cryst Liq Cryst. 256:149.

[2] Neumann F, Teramae H, Downing JW, Michl J (1998) J Am Chem Soc, 120: 573.

[3] Shinjo K (1999) J Jpn Soc Fuzzy Theory Systems, 11 (3):382.

[4] Saito S, Shinjo K, Oida K, Ohtawara K, Shimogawa S, Hirata K, Kitagawa Y, Kuramochi Y, Onda K, Komatsuzaki A, Noguchi T (2000) In Basics and Application of Hamiltonian Algorithm, ATR Technical Report, TR-AC-0049 (in Japanese).

[5] Ohtawara K, Shimogawa S, Shinjo K. (2001) Int J Mod Phys C, 12:1305.

[6] Ohtawara K, Teramae H (2004) Chem Phys Lett 390:84.

[7] Ishimoto T, Tokiwa H, Teramae H, Nagashima U (2004) Chem Phys Lett 387:460-465.

[8] Ishimoto T, H. Tokiwa H, Teramae H,Nagashima U (2005) J Chem Phys 122:094905.

[9] Sato R, Teramae H, Ishimoto T, Nagashima, U (2007) J Comp Chem Jpn 6:295.

[10] Tsuchiya, K, Teramae, H, Watanabe, T, Ishimoto, T, Nagashima U (2007) J Comp Chem Jpn 6: 275-282 (in Japanese).

[11] Teramae H, Ohtawara K, Ishimoto T, Nagashima U (2008) Bull Chem Soc Jpn 81:1094.

[12] Shinjo K, Sasada T (1996) Phys Rev, E54:4685.

[13] Verlet L (1967) Phys Rev 159:98.

[14] Gear CW (1971) Numerical Initial Value Problems in Ordinary Differential Equations, Prentice Hall, Englewood Cliffs, New Jersey.

[15] Schmidt MW, Baldridge KK, Boatz JA, Elbert ST, Gordon MS, Jensen JH, Koseki S, Matsunaga SN, Nguyen KA, Su S, Windus TL, Dupuis M, Montgomery JA (1993) J Comput Chem 14: 1347-1363.

[16] Binkley JS, Pople JA, Hehre WJ (1980) J Am Chem Soc 102:939-47.

[17] Gaussian 03, Revision C.02, Frisch MJ, Trucks GW, Schlegel HB, Scuseria GE, Robb MA, Cheeseman JR, Montgomery Jr. JA, Vreven T, Kudin KN, Burant JC, Millam JM, Iyengar SS, Tomasi J, Barone V, Mennucci B, Cossi M, Scalmani G, Rega N, Petersson GA, Nakatsuji H, Hada M, Ehara M, Toyota K, Fukuda R, Hasegawa J, Ishida M, Nakajima T, Honda Y, Kitao O, Nakai H, Klene M, Li X, Knox JE, Hratchian HP, Cross JB, Bakken V, Adamo C, Jaramillo J, Gomperts R, Stratmann RE, Yazyev O, Austin AJ, Cammi R, Pomelli C, Ochterski JW, Ayala PY, Morokuma K, Voth GA, Salvador P, Dannenberg JJ, Zakrzewski VG, Dapprich S, Daniels AD, Strain MC, Farkas O, Malick DK, Rabuck AD, Raghavachari K, Foresman JB, Ortiz JV, Cui Q, Baboul AG, Clifford S, Cioslowski J, Stefanov BB, Liu G, Liashenko A, Piskorz P, Komaromi I, Martin RL, Fox DJ, Keith T, Al-Laham MA, Peng CY, Nanayakkara A, Challacombe M, Gill PMW, Johnson B, Chen W, Wong MW, Gonzalez C, and Pople JA (2004) Gaussian, Inc., Wallingford CT.

[18] Alexandrova A, Boldyrev A (2005) J Chem Theory Comput 1:566.

[19] Baker J, Wolinski K (2011) J Comp Chem 32:43.

[20] Ohno K, Maeda S (2004) Chem Phys Lett 384:277.

[21] Maeda S, Ohno K (2005) J Phys Chem A 109:5742.

[22] Yang X, Maeda S, Ohno K (2007) J Phys Chem A 111:5099.

[23] Maeda S, Ohno K, Morokuma K. (2009) J Phys Chem A 113:1704. 
[24] Maeda S, Ohno K, Morokuma K. (2009) J Chem Theory Comput 5:2734.

[25] Jalkanen KJ, Suhai S (1996) Chem Phys 208:81-116.

[26] Deng Z, Polavarapu PL, Ford SJ, Hecht L, Barron LD, Ewig CS, Jalkanen KJ (1996) J Phys Chem 100:2025-2034.

[27] Tajkhorshid E, Jalkanen KJ, Suhai S (1998) J Phys Chem B 102:5899-5913.

Han W-G, Jalkanen KJ, Elstner M, Suhai S (1998) J Phys Chem B 102:2587-2602.

[28] Frimand K, Bohr H, Jalkanen KJ, Suhai S (2000) Chem Phys 255:165-194.

[29] Jalkanen KJ, Nieminen RM, Frimand K, Bohr J, Bohr H, Wade RC, Tajkhorshid E, and Suhai S (2001) Chem Phys 265:125-151.

[30] Jalkanen KJ (2003) J Phys Condens Matter 15:S1823-S1851.

[31] Jalkanen KJ, Elstner M, Suhai S (2004) J Mol Struct (Theochem) 675: 61-77.

[32] Jalkanen KJ, Jurgensen VW, Claussen A, Rahim A, Jensen GM, Wade RC, Nardi F, Jung C, Degtyarenko IM, Nieminen RM, Herrmann F, Knapp-Mohammady M, Niehaus TA, Frimand K, Suhai S (2006) Int J Quantum Chem 106:1160.

[33] Jalkanen KJ, Degtyarenko IM, Nieminen RM, Cao X, Nafie LA, Zhu F, Barron LD (2008) Theor Chem Acc 119:191-210.

[33] Deplazes E, Van Bronswijk W, Zhu F, Barron LD, Ma S, Nafie LA, Jalkanen KJ (2008) Theor Chem Acc 119:155-176.

[34] Guerra CF, Van der Wijst T, Poater J, Swart M, Bickelhaupt FM (2010) Theor Chem Acc 125:245-252.

[35] Jankowski K, Nowakowski K, Grabowski I, Wasilewski J (2010) Theor Chem Acc 125:433444.

[36] Van Mourik T, Danilov VI, Dailidonis VV, Kurita N, Wakabayashi H and Tsukamoto T (2010) Theor Chem Acc 125:233-244.

[37] GaussView, Version 5, Dennington R, Keith T, Millam J. (2009) Semichem Inc., Shawnee Mission KS.

[38] Gaussian 09, Revision A.02, Frisch MJ, Trucks GW, Schlegel HB, Scuseria GE, Robb MA, Cheeseman JR, Scalmani G, Barone V, Mennucci B, Petersson GA, Nakatsuji H, Caricato M, Li X, Hratchian HP, Izmaylov AF, Bloino, J, Zheng G, Sonnenberg JL, Hada M, Ehara M, Toyota K, Fukuda R, Hasegawa J, Ishida M, Nakajima T, Honda Y, Kitao O, Nakai H, Vreven T, Montgomery Jr. JA, Peralta JE, Ogliaro F, Bearpark M, Heyd JJ, Brothers E, Kudin KN, Staroverov VN, Kobayashi R, Normand J, Raghavachari K, Rendell A, Burant JC, Iyengar SS, Tomasi J, Cossi M, Rega N, Millam NJ, Klene M, Knox JE, Cross JB, Bakken V, Adamo C, Jaramillo J, Gomperts R, Stratmann RE, Yazyev O, Austin AJ, Cammi R, Pomelli C, Ochterski JW, Martin RL, Morokuma K, Zakrzewski VG, Voth GA, Salvador P, Dannenberg JJ, Dapprich S, Daniels AD, Farkas Ö, Foresman JB, Ortiz JV, Cioslowski J, Fox DJ (2009) Gaussian, Inc., Wallingford CT. 
Table I. HF/3-21G conformational energies and optimized HSD structures of $N^{-}$ acetyl L-histidine $N$-Methyl amide (NALHNMA).

\begin{tabular}{ccccc}
\hline$\phi$ & $\psi$ & $\chi_{1}$ & $\chi_{2}$ & E(Hartree) \\
\hline-84.05 & 66.33 & 48.77 & 68.48 & -712.535583 \\
-114.13 & 42.52 & 56.63 & 74.32 & -712.527921 \\
172.79 & 175.19 & 45.13 & -90.77 & -712.520942 \\
-85.21 & 62.77 & 45.03 & -109.67 & -712.520715 \\
-85.17 & 67.39 & -61.95 & 176.65 & -712.519645 \\
-140.33 & 27.90 & -153.08 & -63.59 & -712.518301 \\
-169.94 & 169.21 & 56.01 & -159.98 & -712.517249 \\
-132.74 & 31.89 & 52.82 & -100.98 & -712.516878 \\
80.11 & 16.35 & -46.41 & 94.15 & -712.516864 \\
74.23 & 170.17 & -52.30 & 89.11 & -712.515626 \\
74.17 & 170.32 & -52.24 & 89.03 & -712.515624 \\
-146.54 & 115.53 & -171.30 & 163.62 & -712.502558 \\
\hline
\end{tabular}


Table II. B3LYP/6-31G** conformational energies and optimized HSD structures of $N$-acetyl L-histidine $N$-Methyl amide (NALHNMA).

\begin{tabular}{ccccc}
\hline$\phi$ & $\psi$ & $\chi_{1}$ & $\chi_{2}$ & E(Hartree) \\
\hline-82.65 & 67.43 & 49.43 & 69.30 & -720.912092 \\
-83.06 & 65.28 & 43.17 & -122.48 & -720.899145 \\
79.17 & -57.09 & -44.57 & 90.88 & -720.899043 \\
-84.42 & 70.37 & -58.20 & 167.67 & -720.898435 \\
-140.76 & 23.17 & -151.09 & -62.81 & -720.897789 \\
-119.39 & 11.85 & 51.35 & -151.12 & -720.895242 \\
77.26 & -47.09 & -48.28 & 88.15 & -720.895126 \\
77.59 & 170.60 & -53.62 & 86.00 & -720.893786 \\
65.64 & -169.89 & -157.43 & -157.15 & -720.889188 \\
\hline
\end{tabular}


Table III. PCM HF/3-21G conformational energies and optimized HSD structures of $N$-acetyl L-histidine $N$-Methyl amide (NALHNMA).

\begin{tabular}{ccccc}
\hline$\phi$ & $\psi$ & $\chi_{1}$ & $\chi_{2}$ & E(Hartree) \\
\hline-135.73 & 29.76 & -155.61 & -61.18 & -712.549353 \\
-149.31 & 147.26 & -179.12 & -97.97 & -712.542333 \\
-142.82 & 46.50 & -158.41 & 108.78 & -712.541972 \\
-143.31 & 47.72 & -158.80 & 105.60 & -712.541805 \\
-135.85 & 67.29 & -167.97 & 97.80 & -712.541449 \\
-87.99 & 126.36 & -177.71 & 70.85 & -712.541433 \\
-98.04 & 119.71 & -177.23 & 73.17 & -712.541119 \\
-121.48 & 100.50 & -178.41 & 88.46 & -712.541067 \\
-89.31 & 128.06 & -178.26 & 68.90 & -712.540871 \\
-77.12 & -46.45 & -170.34 & 168.05 & -712.540469 \\
-139.37 & 112.24 & -175.42 & 174.08 & -712.539660 \\
-144.20 & 128.73 & -177.23 & -144.54 & -712.539586 \\
-69.09 & 150.35 & -176.69 & 64.15 & -712.539158 \\
-139.94 & 39.52 & -159.15 & 166.82 & -712.538970 \\
-106.63 & 15.36 & -155.81 & -157.98 & -712.537020 \\
\hline
\end{tabular}


Table IV. PCM HF/3-21G conformational energies and optimized HSD structures of $N$-acetyl L-histidine $N$-Methyl amide (NALHNMA) with four water molecules.

\begin{tabular}{ccccc}
\hline$\phi$ & $\psi$ & $\chi_{1}$ & $\chi_{2}$ & E(Hartree) \\
\hline-95.37 & 148.56 & 48.93 & 54.03 & -1015.038309 \\
-157.62 & 160.62 & 51.60 & 82.58 & -1015.038105 \\
-78.17 & 150.82 & 48.97 & 49.14 & -1015.038062 \\
-157.37 & 160.43 & 52.20 & 83.58 & -1015.037978 \\
-141.35 & 128.01 & 42.12 & 46.82 & -1015.037814 \\
-158.12 & 160.35 & 51.80 & 83.05 & -1015.037786 \\
-148.81 & 132.15 & 43.05 & 45.29 & -1015.037675 \\
-159.06 & 158.97 & 49.56 & 83.38 & -1015.037336 \\
-121.79 & 153.02 & 52.80 & 88.56 & -1015.037050 \\
-159.00 & 158.55 & 49.98 & 83.62 & -1015.037008 \\
-139.16 & 113.49 & -17.63 & 86.55 & -1015.036189 \\
-158.83 & 157.43 & 46.48 & 84.43 & -1015.035796 \\
-126.00 & 146.96 & 49.78 & 94.86 & -1015.035458 \\
-79.59 & 151.68 & 50.35 & 42.47 & -1015.033529 \\
-158.14 & 160.29 & 43.39 & 49.36 & -1015.030476 \\
-72.67 & 152.16 & 43.73 & 41.49 & -1015.030029 \\
-71.06 & 152.29 & 43.88 & 41.60 & -1015.030022 \\
-157.43 & 158.84 & 46.02 & 42.32 & -1015.028246 \\
-157.01 & 155.94 & 45.92 & 38.39 & -1015.028189 \\
-64.21 & 149.57 & 46.15 & 48.42 & -1015.025707 \\
-110.69 & 101.02 & -17.62 & 89.32 & -1015.022320 \\
\hline
\end{tabular}


Table V. PCM B3LYP/aug-cc-pVDZ conformational energies and optimized HSD structure of $N$-acetyl L-histidine $N$-Methyl amide (NALHNMA) with four water molecules, optimized from the structures corresponding to first five column of Table IV.

\begin{tabular}{ccccc}
\hline$\phi$ & $\psi$ & $\chi_{1}$ & $\chi_{2}$ & E(Hartree) \\
\hline-138.61 & 144.26 & 50.95 & 87.54 & -1026.845976 \\
-138.72 & 144.06 & 50.78 & 87.56 & -1026.845847 \\
-142.80 & 114.46 & 42.85 & 53.93 & -1026.844829 \\
-96.15 & 139.07 & 52.08 & 61.31 & -1026.844662 \\
-96.02 & 139.25 & 52.11 & 61.31 & -1026.844661 \\
\hline
\end{tabular}




\section{Figure Captions}

Figure 1. Plots of distribution of eigenvalues when mixing coefficient is $\Delta$. The fitting functions used are none (i.e., without mixing), linear, quadratic, and quartic functions.

Figure 2. Plots of dihedral angles of C-C-C-C backbone in butane molecule for first 2000 iterative HA calculations using distribution of eigenvalues with (a) linear, (b) quadratic, and (c) quartic functions, using mixing coefficients 0.0 (i.e., without mixing), 0.05 and 0.1 , respectively.

Figure 3. Plots of dihedral angles with full 60000 iterative HA calculations (a) without mixing and with mixing with (b) linear, (c) quadratic, and (d) quartic functions. The mixing coefficient is kept at 0.15 except for the case without mixing.

Figure 4. $N$-acetyl L-histidine $N$ '-Methyl amide and four water molecules, PCM B3LYP/aug-ccpVDZ optimized structures. (a)-(e) correspond to 1-5 columns of Table V. 


\section{Distribution of eigenvalues}

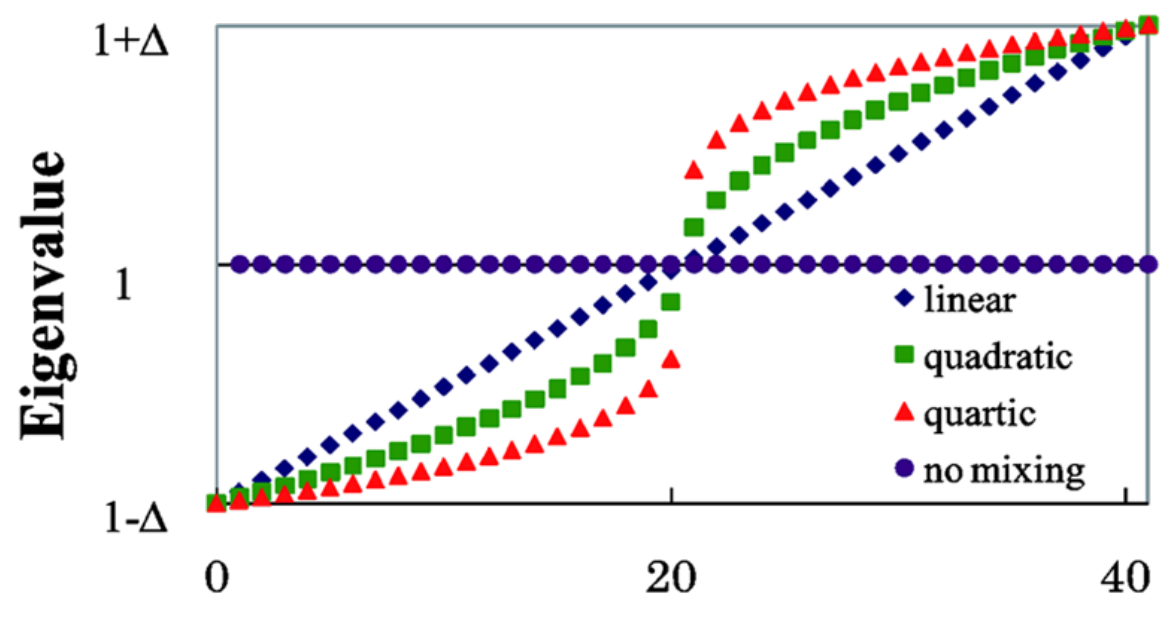

Coordinate number

Figure 1 Teramae et al.

(a) Linear function

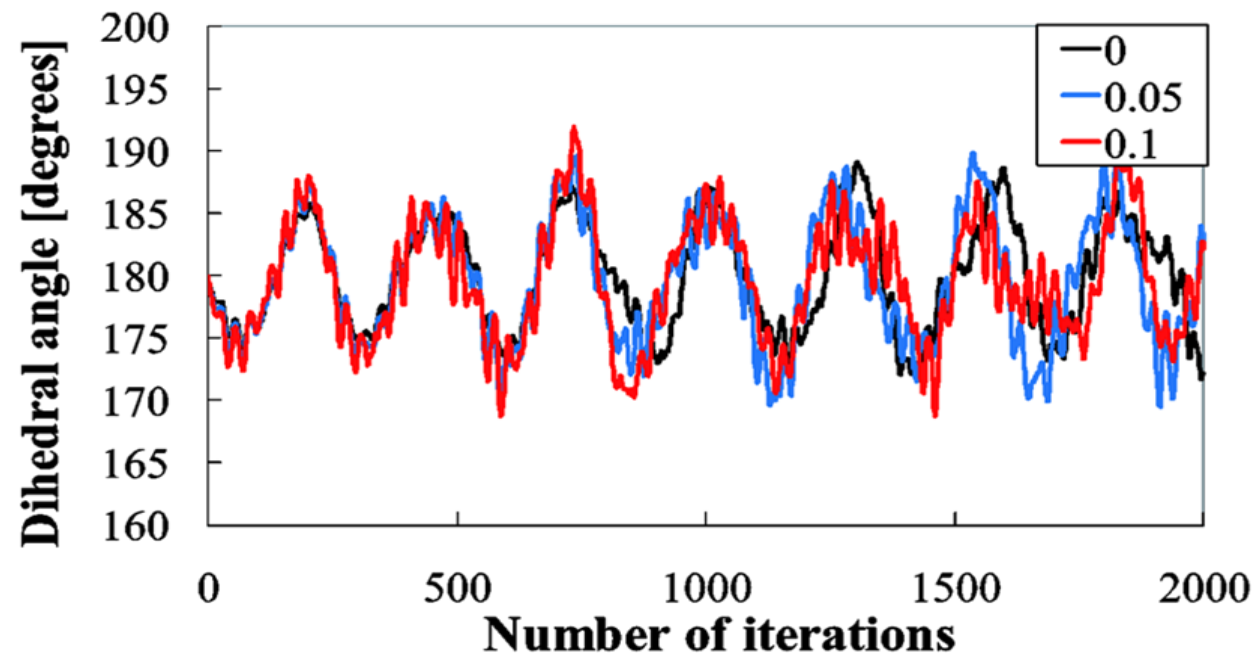

Figure 2(a) Teramae et al. 
(b) Quadratic function

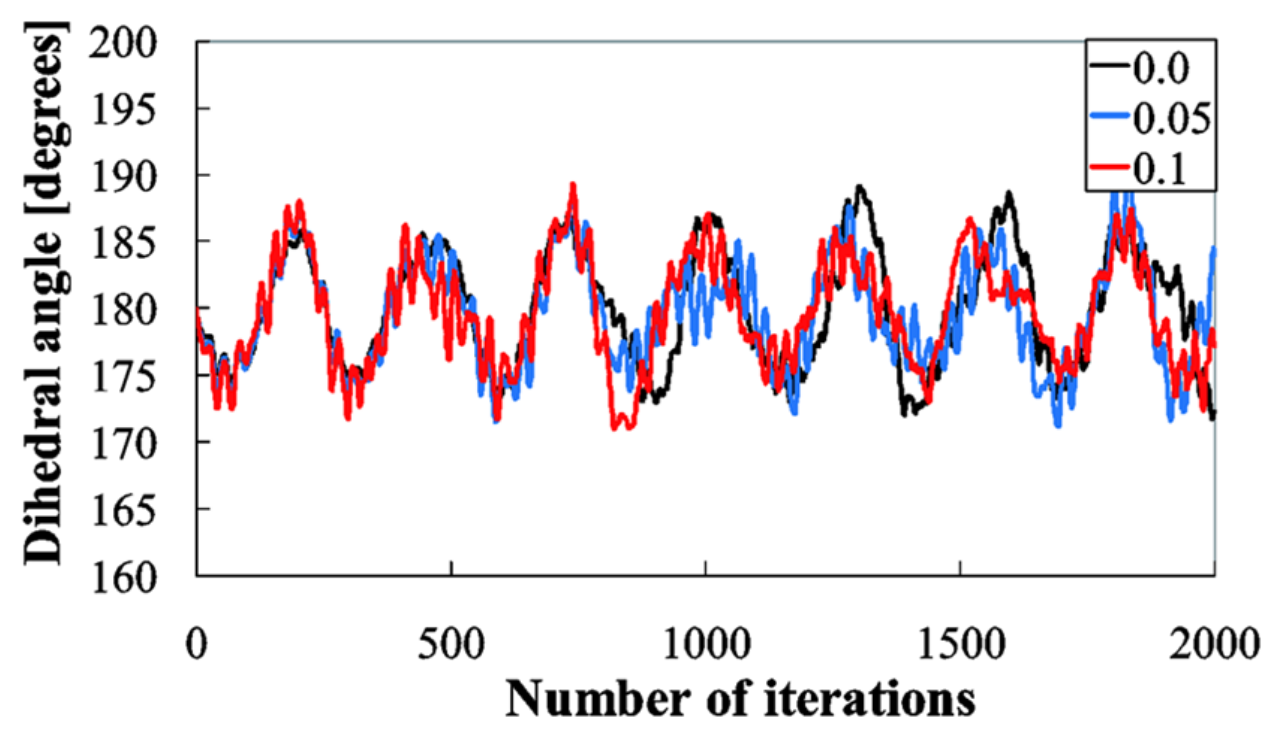

Figure 2(b) Teramae et al.

(c) Quartic function

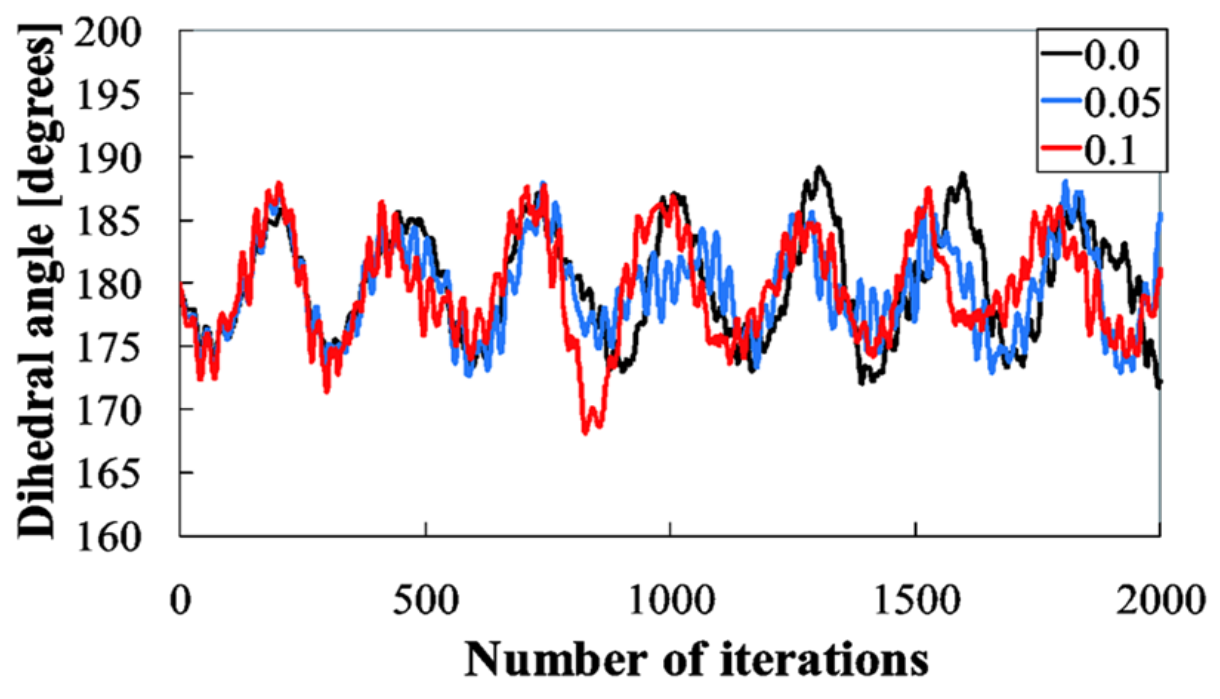

Figure 2(c) Teramae et al. 
(a) Without mixing

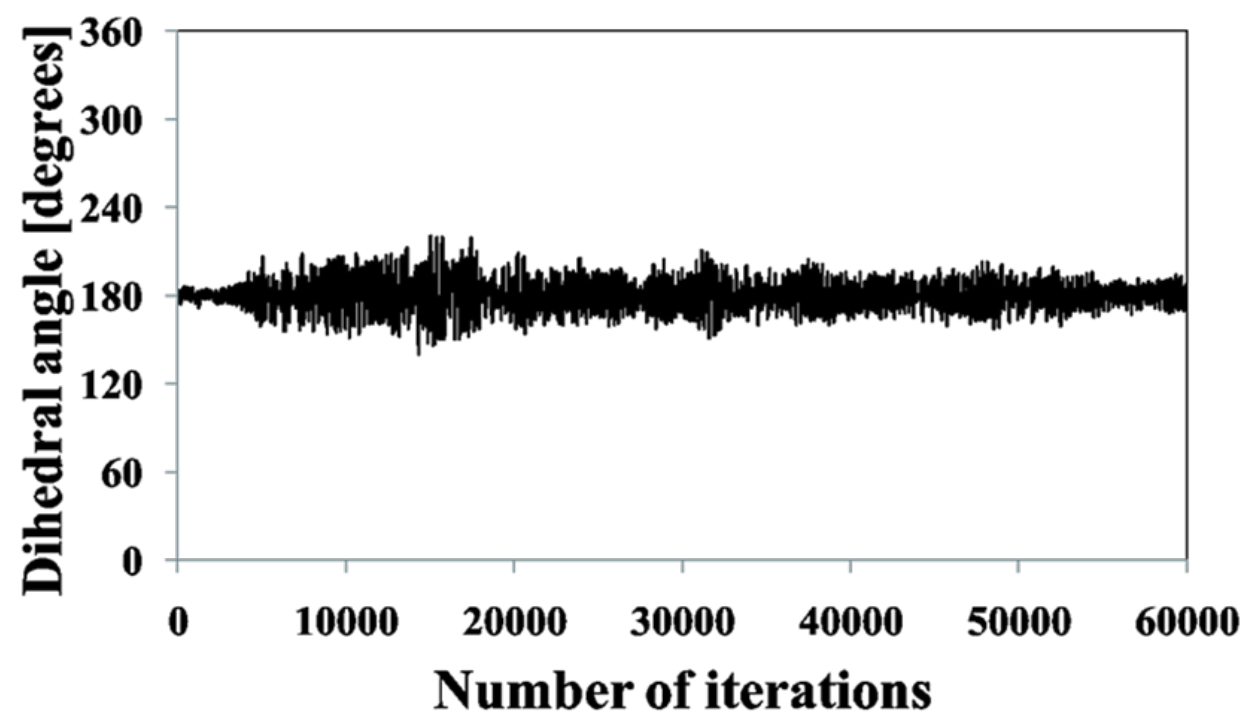

Figure 3 (a) Teramae et al.

(b) Linear function

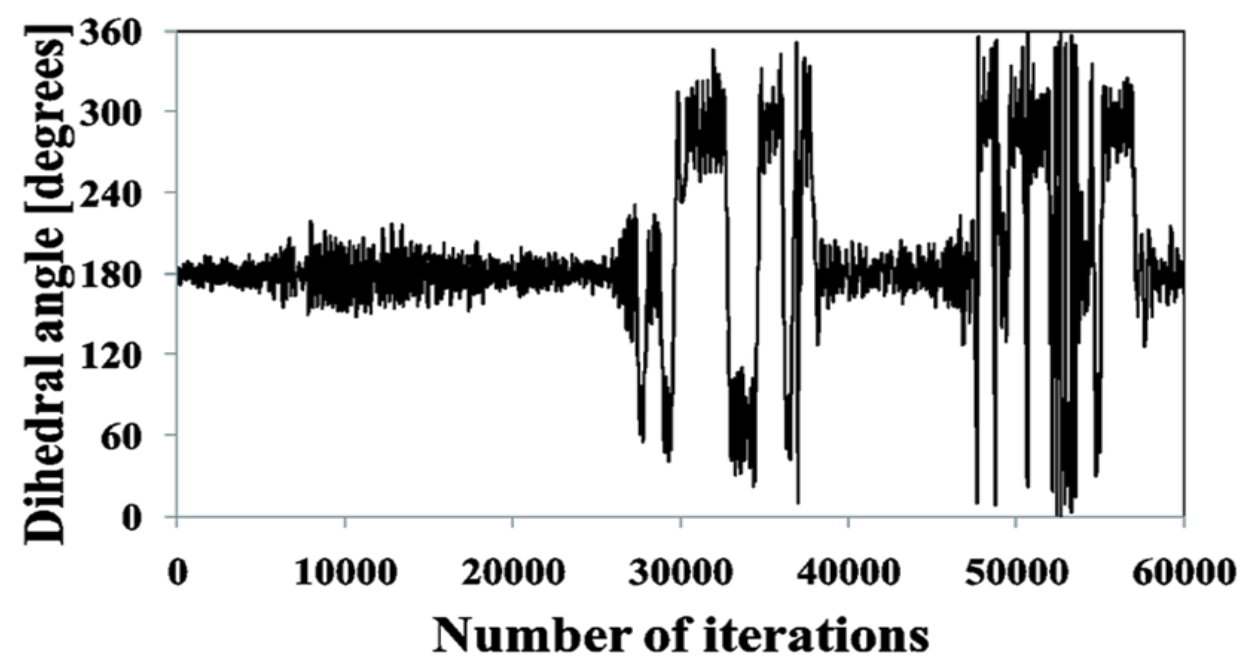

Figure 3 (b) Teramae et al. 
(c) Quadratic function

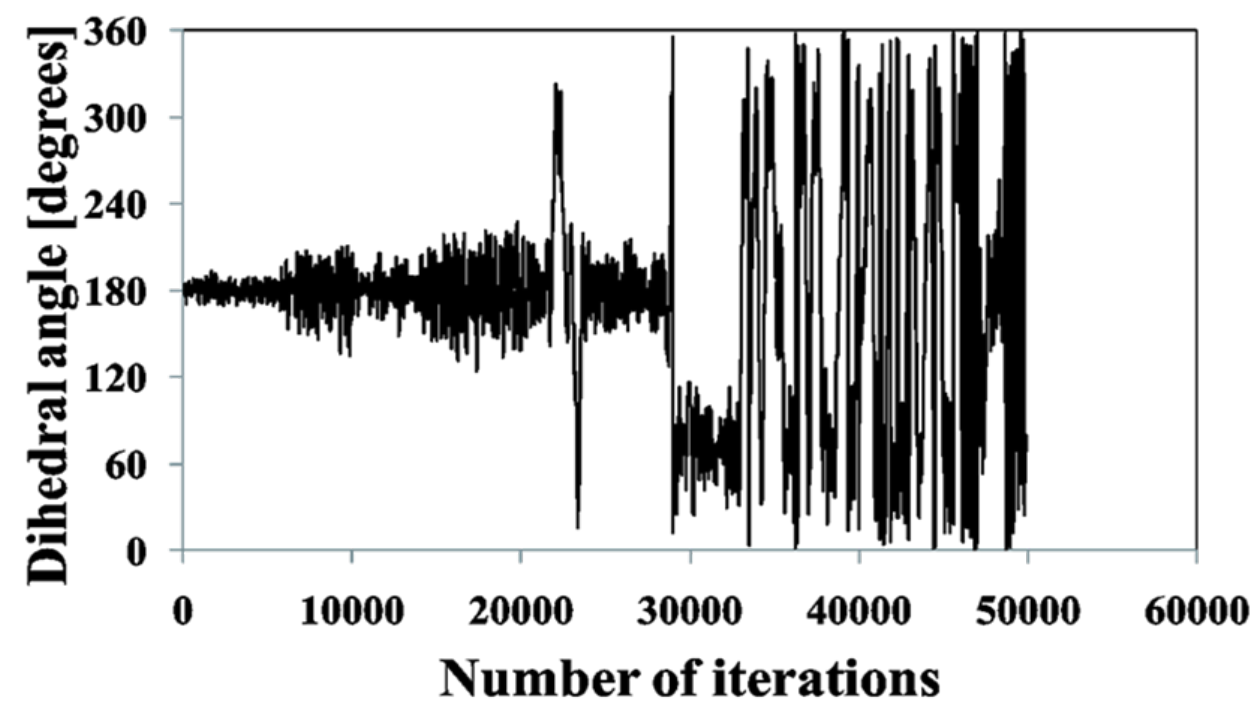

Figure 3 (c) Teramae et al.

(d) Quartic function

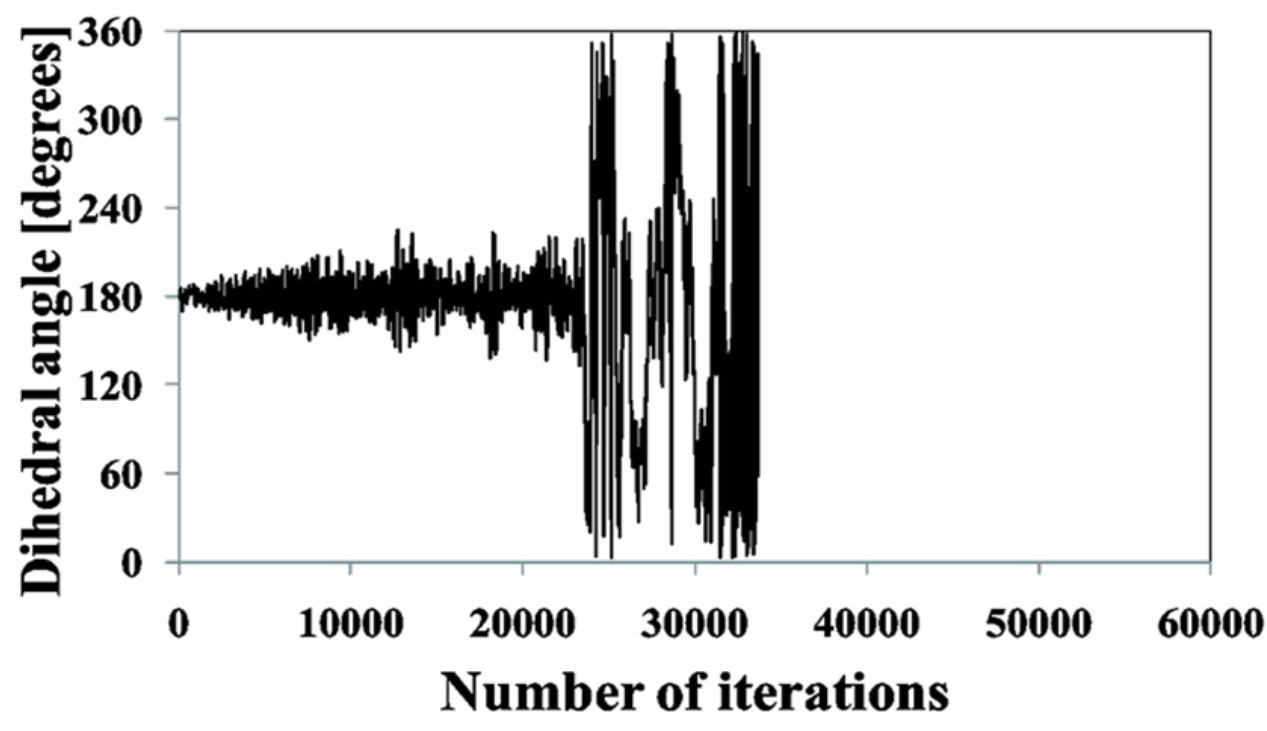

Figure 3 (d) Teramae et al. 


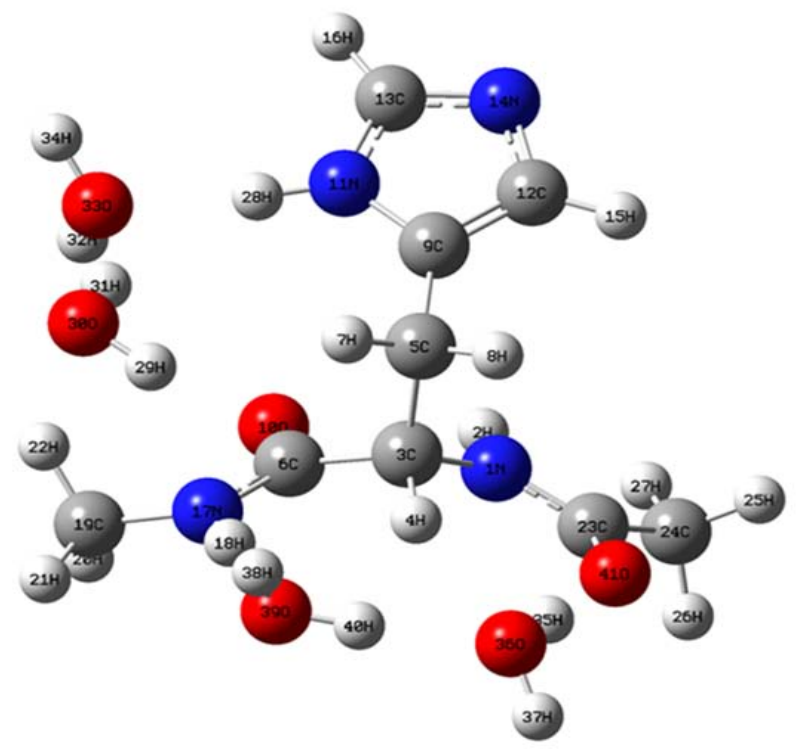

Figure 4 (a) Teramae et al.

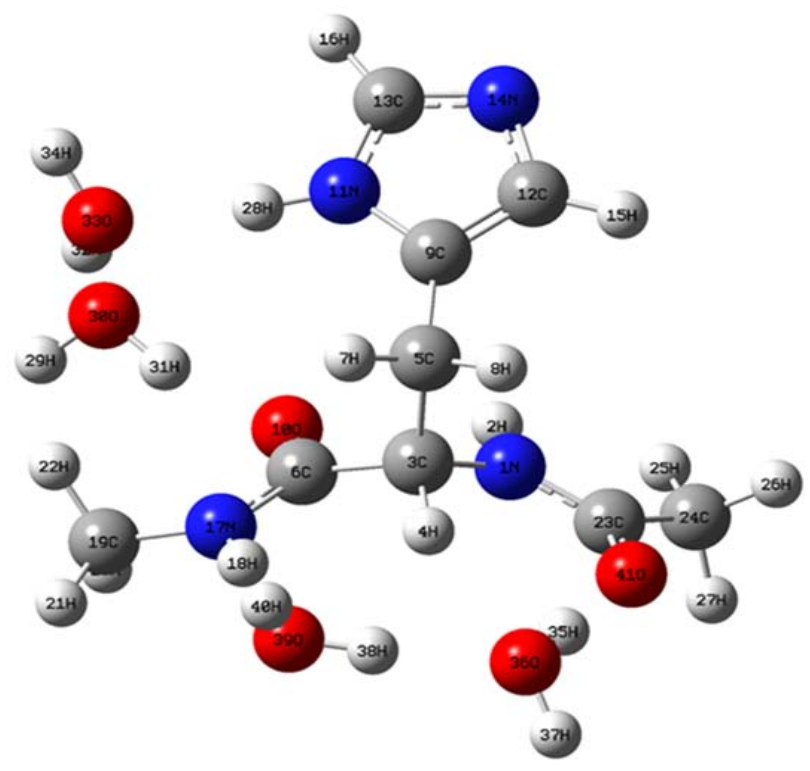

Figure 4 (b) Teramae et al. 


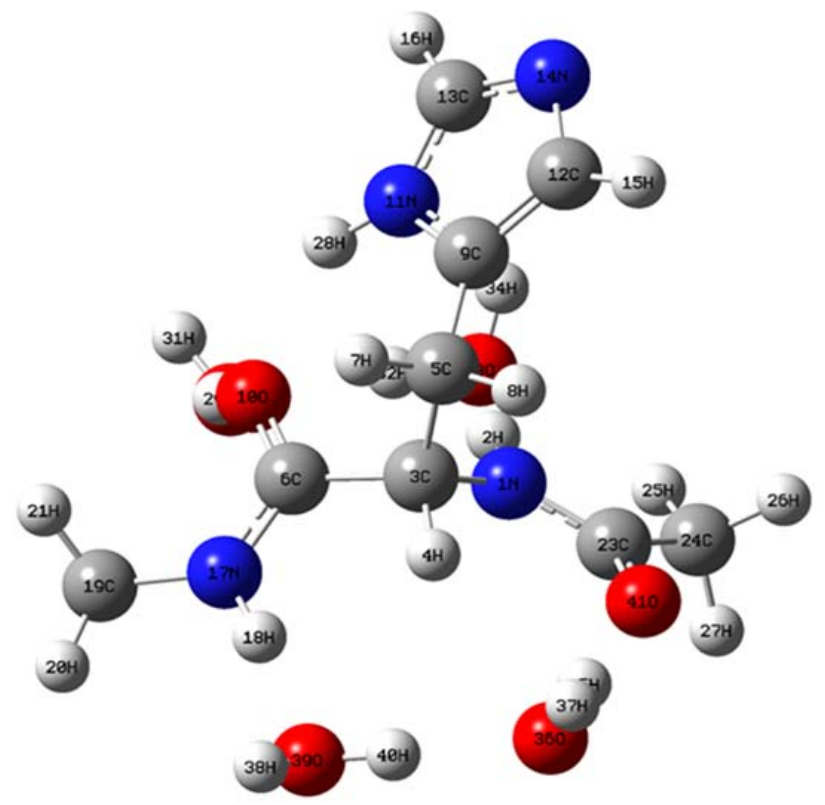

Figure 4 (c) Teramae et al.

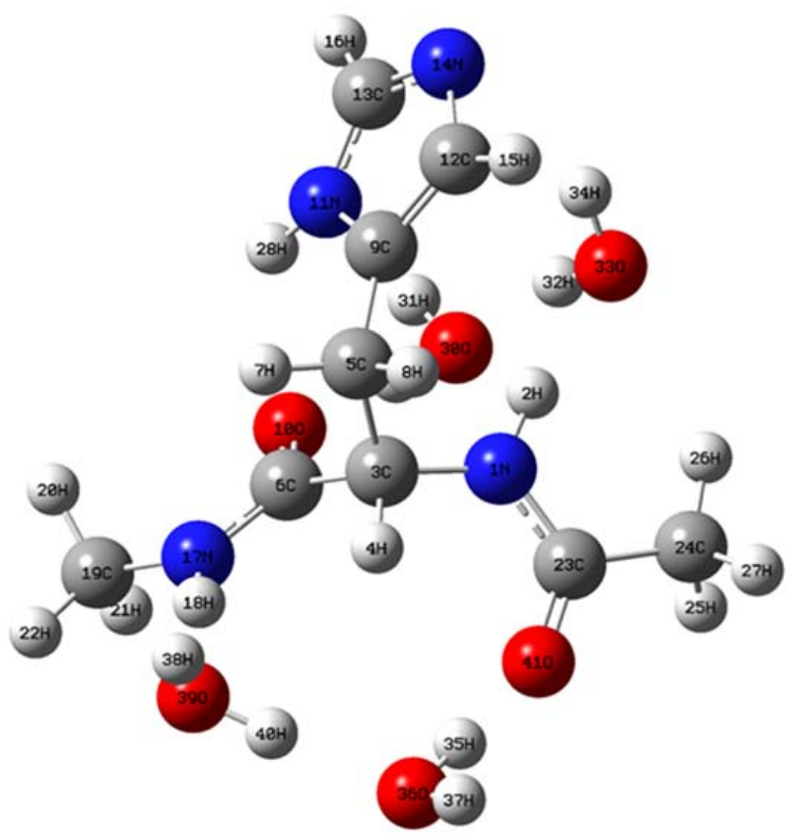

Figure 4 (d) Teramae et al. 


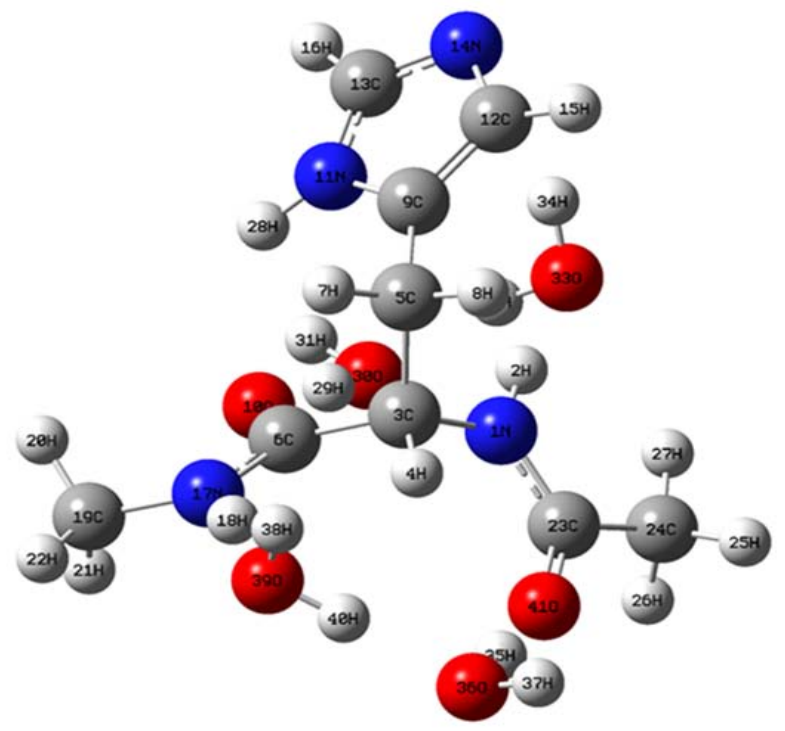

Figure 4 (e) Teramae et al. 